\title{
Los Estudios de Opinión y su Influencia en las Preferencias de las Personas
}

\section{The Influence of Opinion Polls on People's Preferences}

\author{
Rodrigo Uribe \\ Enrique Manzur \\ Universidad de Chile
}

\begin{abstract}
Este artículo revisa la evidencia existente sobre la influencia que las encuestas tienen en la manifestación de preferencias tanto en términos de asumir una posición (de mayoría - bandwagon-o de minoría -underdog) como de las variables que mediatizan este efecto. A continuación se presenta un estudio experimental que analiza este último punto en relación a dos variables de las encuestas (dirección y fuerza de los resultados) y tres elementos individuales (personalidad, estilo cognitivo y género). Los resultados señalan que la fuerza de la encuesta así como un mayor puntaje en la escala de extroversión y neuroticismo (medido a través del EPI) y el género (masculino) efectivamente están asociados a la presencia del efecto bandwagon.
\end{abstract}

Palabras Clave: persuasión, personalidad, “need for cognition”, encuestas, efecto "bandwagon", efecto "underdog”.

\begin{abstract}
This article develops a literature review of the evidence concerning the effects of opinion polls on people's preferences (in the direction of the majority - bandwagon- or minority of people - underdog) as well as the variables that moderate the presence of this effect. Secondly, an experimental study is reported. It analyzes two variables related to the nature of opinion polls (direction and strength of poll results) in terms of their effect on preferences change. Finally, it analizes the role of three individual factors (personality, cognitive style, and gender), which are correlated with the presence of this effect. Results show that strength of poll results, as well as gender (male) and a higher score on extroversion and neuroticism (measured with tuhe EPI), are actually associated with the bandwagon effect.
\end{abstract}

Keywords: persuasion, personality, need for cognition, polls, bandwagon efect, underdog effect.

¿Votaría por alguien o algo que la mayoría de la gente señala no preferir? Probablemente lo pensaría más de una vez. Basados en que la opinión grupal puede resultar significativa a la hora de explicar algunas de nuestras preferencias personales, el desarrollo de estrategias persuasivas ha regularmente contemplado la difusión de estudios de opinión como herramienta de influencia sobre el grupo destinatario (Cialdini, 1993). Un ejemplo de lo anterior son las campañas políticas, las cuales recurrentemente dan a conocer resultados de encuestas favorables (no importando si éstas son metodológicamente sólidas), intentando crear en la opinión pública la percepción que su candidato o postura es la ganadora o, al menos, que va en

Rodrigo Uribe B., Departamento de Administración, Facultad de Economía y Negocios, Universidad de Chile.

Enrique Manzur M., Departamento de Administración, Facultad de Economía y Negocios, Universidad de Chile.

La correspondencia relativa a este artículo deberá ser dirigida a Rodrigo Uribe y/o Enrique Manzur a Diagonal Paraguay 257, Santiago, Chile. E-mail: ruribe@unegocios.cl, emanzur@, unegocios.cl

Los autores desean reconocer y agradecer el trabajo de Bárbara García y Valeria Valdebenito en la recolección y análisis de datos. alza en la aprobación popular ${ }^{1}$. Ello, con el objetivo que las personas que no son afines del todo a esta posición cambien su preferencia a través de sumarse a la tendencia mayoritaria (Maarek, 1997; Noelle-Neumann, 1984).

Al respecto, un largo cuerpo de investigaciones ha analizado la influencia que las encuestas pueden llegar a tener en las opiniones que las personas expresan. Dichos estudios han intentado aclarar si la presentación de estudios de opinión hace o no a las personas directamente variar su postura $y$, como consecuencia, sumarse a la creencia mayoritaria -efecto bandwagon- o, en algunos casos, a la opinión de minoría -efecto underdog (ver, por ejemplo, Crespin \& Wielen, 2002; Fleitas, 1971; Simon, 1954; Sudman, 1986). La evidencia respecto de estos temas no ha mostrado resultados del todo concluyentes, aunque ha tendido a dar mayor soporte a la idea que los estudios de opinión pueden llegar a ser una herramienta relevante de persuasión, espe-

\footnotetext{
1 En ese contexto, resulta ilustrativo mencionar los hallazgos de Steihorn (2000) en los EEUU, quien reporta que en el período previo a la elección del año 2000 , los medios noticiosos presentaron cinco veces más encuestas que en el período preeleccionario de 1980.
} 
cialmente en la dirección de la opinión mayoritaria o bandwagon (Marsh, 1984).

De modo complementario, otro número importante de estudios ha intentado determinar cuáles son las condiciones en las cuales los estudios de opinión pueden llegar a tener un efecto significativo. Dicho de otro modo, se ha tratado de identificar qué variables aparecen como mediadoras del potencial efecto (amplificándolo o aminorándolo) que tienen las encuestas en sus receptores (Areni, Ferrell \& Wilcox, 1998; Lang \& Lang, 1984). Al respecto se ha explorado el rol que tiene una serie de elementos tanto de las audiencias como del estímulo (encuestas) de modo de determinar algunos de los factores relevantes que ayudan a incrementar o diminuir la persuasividad de los estudios de opinión (Areni, Ferrell \& Wilcox, 2000).

En el contexto de esta última línea de investigación, el presente artículo reporta un estudio experimental que explora algunas variables mediacionales del potencial efecto persuasivo de las encuesta. En concreto, se analizan variables tanto de la encuesta, como la dirección de los resultados (a favor o en contra de lo preguntado) y su fortaleza (diferencia porcentual entre las preferencias por una postura y otra), así como de los receptores como son el tipo de personalidad, el estilo cognitivo y el género del receptor.

\section{¿Qué Influencia Pueden Tener los Estudios de Opinión?}

Diversos autores han sostenido que las encuestas no deberían ser consideradas meramente portavoces de la opinión mayoritaria, sino que también potenciales conductores de la opinión de la mayoría. Es decir, los estudios de opinión no sólo tenderían a mostrar la visión de las personas, sino que las opiniones de las personas también pueden estar reflejando los resultados de dichos estudios (Morwitz \& Pluzinski, 1996). Basado en esos principios, algunos países como los EE.UU. y Canadá han llegado incluso a regular la presentación de estudios de opinión antes y durante cualquier período eleccionario como medio de evitar su influjo sobre la opinión pública (Andersen, 2000; Donsbach, 2001; Lachapelle, 1991).

La influencia que los estudios de opinión pueden llegar a tener se ha establecido en términos de dos efectos opuestos: bandwagon y underdog. El primero, que es el más frecuentemente señalado en la literatura, sostiene que los estudios de opinión ejercerían una función similar a la llevada a cabo por el carro de un tren que arrastra a los otros. En el contexto de la opinión pública y la persuasión, este efecto se manifestaría en que el público tenderá a manifestar una opinión o intención de voto proclive por la posición que aparece como mayoritaria. De esta forma, si una persona ve -a través de un estudio de opinión- que la mayoría tiende a apoyar a una persona o idea por sobre otra, ésta debería seguir la tendencia colectiva y eventualmente cambiar su postura original (Marsh, 1984; Mehrabian, 1998; Navazio, 1977).

Por su parte, el fenómeno opuesto ha sido denominado efecto underdog. Este plantea que, producto de ser expuestas a la opinión grupal, algunas personas mostrarían la tendencia contraria a aquella descrita por el efecto bandwagon. Esta influencia se manifestaría en una simpatía hacia la posición minoritaria exhibida por las encuestas (Lavrakas \& Traugott, 2000). De esta forma, la información de los estudios de opinión produciría una influencia de desaliento de la posición mayoritaria del grupo $y$, por contraste, facilitaría el adoptar una posición de minoría que, sin la presencia de los estudios, no se hubiese asumido necesariamente (Fleitas, 1971; Gartner, 1976).

\section{¿Qué Evidencia Existe Acerca de la Presencia de Efectos de los Estudios de Opinión?}

A la fecha, los estudios han provisto evidencia que no es del todo conclusiva acerca de la existencia de un efecto de los estudios de opinión sobre la manifestación de preferencias. En la literatura se encuentran un número significativo de investigaciones que dan soporte, pero así también otras que no dan apoyo a la tesis de la existencia de una influencia ya sea en la dirección propuesta por el efecto bandwagon o el underdog (Marsh, 1984).

Por un lado, existe un grupo de estudios que tiende a ser superior en número que ha probado la ocurrencia de algún efecto significativo producido por la presentación de encuestas. La mayor parte de ellos han demostrado la existencia de una tendencia a sumarse a la opinión mayoritaria (bandwagon). $\mathrm{Al}$ respecto, un estudio desarrollado por White (1975) presentó estudios de opinión a diversos grupos de participantes, observando que cuando los resultados eran más extremos se producían cambios más radicales en la opinión de las personas. En un estudio más reciente, Mehrabian (1998) analizó experimentalmente el comportamiento de votantes republicanos de Iowa (EE.UU.) cuatro días antes de la elección 
primaria de ese partido en 1996. Se pudo observar una tendencia significativa de votar por el candidato que aparecía liderando las encuestas, comprobando el efecto bandwagon, el cual explicaba un $6 \%$ de la varianza en las preferencias manifestadas por las personas que participaron del estudio (ver también Ansolabehere \& Iyengar, 1993; Bartels, 1985; Cloutier, Nadeau \& Guay, 1989; Fleitas, 1971; Goidel \& Shields, 1996; Kaplowitz, Fink, D’Alessio \& Armstrong, 1983; Roshwalb \& Resnicoff, 1971; Schmitt-Beck, 1996; Skalaban, 1988; West, 1991).

Dentro de los estudios que han probado la existencia de un efecto de la exposición a estudios de opinión, existe un número menor que ha logrado mostrar el efecto opuesto, es decir, underdog. Por ejemplo, Lavrakas, Holley y Miller (1991) usaron una muestra nacional en EE.UU. para testear la presencia de algún efecto de los estudios de opinión durante la elección de 1988. A la mitad de la muestra le señalaron qué candidato dominaba en las encuestas. Usaron análisis discriminante para predecir por qué candidato esas personas hubiesen votado si no hubiesen tenido esa información. Si bien ellos observaron una influencia en la dirección predicha por tanto en efecto bandwagon como por el underdog, la presencia del segundo de éstos llegó a ser tres veces superior (ver también McAllister \& Studlar, 1991; Tangeras, 1998).

Por otro lado, existe un número de estudios que han rechazado la hipótesis de un efecto generado por la presentación de estudios de opinión. Por ejemplo, Mendelson (1966) examinó experimentalmente si el efecto que la información transmitida por el noticiario en TV acerca del resultado electoral en un extremo este de los EE.UU. tenía efecto en los electores de California, no observando evidencia acerca de algún efecto bandwagon o underdog en los votantes de la costa Oeste (ver también Ceci \& Kain, 1982; Marsh, 1984; Navazio, 1977).

La existencia de resultados que presentan cierta contradicción ha sido explicada principalmente por la naturaleza de los efectos de la comunicación persuasiva, la cual muchas veces opera mediada por otros factores. Desde los estudios clásicos de Katz y Lazarrsfeld (1955) se ha sostenido reiteradamente que el efecto de la persuasión puede actuar muchas veces tanto en procesos directos como indirectos (Wolf, 1994). Estos últimos son los modelos de dos o tres pasos, en que el efecto es mediatizado por variables tanto del mensaje (por ejemplo el medio de comunicación en que se transmite), del individuo receptor (su personalidad o creencias previas), como del contexto social (líderes de opinión y la agenda de los medios de comunicación) (ver, por ejemplo, Mc Quail, 2000; Mutz, 1998). De esta forma, aquellos estudios que han privilegiado la medición directa del efecto de las encuestas han observado sólo en ocasiones diferencias significativas (Diaz-Castillo, 2005).

\section{¿Qué Factores Condicionan la Presencia de un Efecto de las Encuestas en la Manifestación de Preferencias?}

En este contexto, una pregunta cada vez más reiterada en los estudios acerca de la influencia de las encuestas es cuáles son aquellos elementos que pueden actuar aumentando la probabilidad de una mayor presencia del efecto bandwagon o underdog. Al respecto, la investigación previa ha establecido una serie de factores tanto propios de los estímulos como individuales que pueden actuar mediatizando el efecto de los estudios de opinión sobre quienes los reciben (Areni et al., 2000).

En relación a factores asociados al estudio mismo, la investigación previa ha provisto evidencia de al menos cuatro elementos significativos los cuales sugieren que cuando el estímulo se asocia a un menor involucramiento y tiende a favorecer un procesamiento de la información más bien basado en claves accesorias (procesamiento periférico) que lógicas (procesamiento centralizado), los estudios de opinión tenderían a ejercer una mayor influencia.

Al respecto, un primer elemento de los estudios de opinión señalado como mediador de su influencia es la importancia del tópico preguntado. Areni y colaboradores (1998) examinaron en Australia cómo la mayor o menor relevancia asignada a un tema podría estar asociada al efecto de los estudios de opinión. Ellos pudieron comprobar que en aquellos temas que las personas evaluaban como secundarios, la opinión grupal era utilizada como una clave muy importante a la hora de asumir una postura, lo cual no ocurría en asuntos que generaban un mayor involucramiento (ver también, Lang \& Lang, 1984). Un segundo factor relevante se refiere al tipo de votación realizada (referéndum de apoyo o rechazo a una opción versus elección entre diferentes alternativas). Al respecto West (1991) analizó comparativamente si la exposición a encuestas influenciaba la decisión de voto en un contexto de elección (presidencial de los EE.UU. de 1980) y de referéndum (sobre el derecho a la vida realizado en 1986 en un estado de los EE.UU.). Dicho autor pudo detectar la presencia del 
efecto bandwagon sólo en el segundo de los casos y no en el primero, lo cual fue atribuido a que en un referéndum usualmente se tratan temas de menor relevancia que en las elecciones, por lo que las opiniones de los otros aparecen como un elemento de mayor importancia en la preferencia. En tercer lugar, Fleitas (1971) incorpora otro elemento que puede actuar como mediador: una elección local versus una nacional. Al comparar las preferencias acerca de una elección local y los de una de nivel nacional, dicho autor pudo detectar que en el primero de los casos los electores tendían a ser más influenciados por los estudios de opinión (particularmente en la dirección bandwagon), lo cual fue explicado porque en ellas existiría un menor involucramiento de los electores lo que acrecienta el rol de los otros en la construcción de la opinión. Finalmente, Mehrabian (1998) estableció que la fuerza de los resultados de la encuesta (distancia entre una posición y otra) podía aumentar el efecto del estudio de opinión sobre las preferencias personales, ello principalmente porque resultados menos estrechos tendían a ser leídos por las personas como una manifestación clara acerca de una posición como correcta (ver también White, 1975).

Por otro lado, la literatura también ha provisto evidencia sobre variables relevantes de las audiencias de los estudios de opinión. Una de ellas se obtiene del mencionado estudio publicado en 1971 por Fleitas donde dicho autor pudo observar que en un contexto de menor cantidad de información poseída por la persona (que significa que la persona sabe qué resultado es más probable), un importante número de votantes tendían a variar sus preferencias. Contrariamente, en contextos en que las personas están más informadas, el efecto de los estudios de opinión tendía a ser menos significativo ya que serían considerados como una clave relevante. Un segundo factor relevante es proporcionado por Marsh (1984) en Gran Bretaña, quien reporta que los grupos que han recién comenzado su participación en votaciones (cercanos a los 18 años) poseen una mayor susceptibilidad a ser afectados por la presentación de encuestas, dado que poseen una menor fortaleza de la opinión. Finalmente, Mehrabian (1998) reporta un estudio experimental que examina la presencia del efecto bandwagon en relación a diferentes estilos de personalidad. Dicho autor pudo observar que aquellos perfiles con una mayor tendencia a la inestabilidad y excitabilidad frente a estímulos externos, así como a tener un temperamento más influenciable y abierto a lo externo (extroversión) tienden a ser más influenciados por los estudios de opinión (ver resultados similares para publicidad en Mooradian, 1996).

\section{Método}

En el contexto de los antecedentes previamente expuestos, se desarrolló un estudio experimental que buscó examinar el rol de algunas variables que median en el efecto que los estudios de opinión tienen en las personas, replicando parcialmente y buscando expandir una de las exploraciones más recientes en el área (Mehrabian, 1998). En concreto, se examinó el rol que poseen dos factores asociados a la encuesta y tres relacionados con los receptores. Respecto de aquellos relacionados con la encuesta, se analizaron la fuerza de los resultados y la dirección de ésta (que en el estudio de Mehrabian de 1998 no había mostrado tener un rol relevante). Adicionalmente, se estudiaron algunas características individuales. Una de ellas fue la personalidad (usando el test EPI) básicamente buscando re-explorar los hallazgos de Mehrabian (1998) así como Mooradian (1996) en la línea que estilos de personalidad más extrovertidos y con un más alto neuroticismo deberían ser más responsivos a las encuestas. Simultáneamente, se evaluó la existencia de diferencias según el tipo de procesamiento de la información. Asumiendo que un procesamiento más centralizado debería limitar la influencia de las encuestas en comparación con uno más periférico, se indagó la presencia diferencial de cambios en el sentido del efecto bandwagon usando un test de estilo cognitivo (el test Need for Cognition). Este análisis deriva de la hipótesis de Areni et al. (1998) quienes plantean la potencial mayor probabilidad de influencia de los estudios de opinión en un contexto de procesamiento periférico, lo cual no lograron probar en sus estudio.

\section{Diseño}

Ciento treinta y dos alumnos universitarios de pregrado ( 75 hombres y 55 mujeres) participaron voluntariamente en este experimento. Se utilizó una pregunta de tipo referéndum acerca si deseaban la mantención del sistema actual de fijación de pruebas imperante en la Facultad (semana de pruebas) en las que los participantes del estudio son alumnos. Se pidió a los participantes del estudio que manifestaran su preferencia luego de ser expuestos a una encuesta que contenía una de las cuatro manipulaciones usadas. El experimento - un $2 \times 2$ between subject-, incorporaba dos factores con dos valores cada uno: dirección (favor o en contra de la semana de pruebas) y fuerza de la encuesta (moderadamente o fuertemente a favor o en contra de la semana de pruebas).

A continuación se midieron las diferencias en la sensibilidad a los estudios de opinión usando un test de personalidad y uno de estilo cognitivo. Para el primero, se empleó el Inventario de Personalidad de Eysenck-EPI- (Eysenck \& Eysenck, 1964) que comprende 57 ítemes, 24 de ellos midiendo extroversión, 24 introversión y los restantes 9 conformando una escala de veracidad (también llamada sinceridad, que busca establecer la presencia de deseabilidad social en las respuestas). Eysenck plantea que la personalidad está constituida por un número de dimensiones. En la versión del test aplicado (EPI) se diferencian dos (neuroticismo e introversión), aunque posteriormente este mismo autor incorpora una tercera (psicoticismo) a su teoría (Eysenck, 1992). En lo que se refiere a la dimensión neuroticismo, se presenta una oposición que tiene como polo contrario la estabilidad. Un alto 
grado de neuroticismo se vincula con una baja tolerancia para el estrés y dolor (sea éste físico o psicológico) y, en general, con labilidad emocional e hiperactividad (Eysenck, 1990, 1991). Por su parte, la dimensión extroversión- introversión se suele manifestar en la tendencia a ser expansivos, con numerosos contactos sociales y actividades grupales, así como búsqueda de sensaciones intensas.

Para el análisis del estilo cognitivo se utilizó el test Need for Cognition (Petty \& Cacioppo, 1986a), el cual examina el grado de disfrute que las personas poseen desarrollando actividades que implican un esfuerzo cognitivo. Este test está basado en el modelo ELM (Elaboration Likelihood Model, Petty \& Cacioppo, 1986a, 1986b), que caracteriza la persuasión operando por la acción dominante de una de dos rutas que se pueden ubicar en un continuo que va desde el procesamiento centralizado al periférico. El primero de éstos ocurre cuando las personas realizan una consideración detallada de la lógica y validez de la información presentada. Por su parte el segundo se refiere al uso de claves secundarias (tales como perceptuales, genéricas, emocionales) como elementos que hacen procesar el estímulo (Haugtvedt, Petty \& Cacioppo, 1992). El uso de una u otra ruta está motivado por diferentes factores. Por un lado están los factores del estímulo, donde claramente ciertos contenidos poseen una mayor tendencia social que otros a ser procesados de determinada forma (por ejemplo, un tema de alta relevancia social tiende a ser analizado más centralizadamente). Por otro lado, están los factores de las personas, donde hay elementos situacionales (por ejemplo, nivel de energía) y de disposición. A este último nivel es donde se ha señalado que existe una tendencia de ciertas personas a procesar estímulos preferentemente usando una u otra ruta. De esta forma, el test Need for Cognition ha sido desarrollado para dar cuenta de estas diferencias individuales disposicionales (Areni et al., 1998).

\section{Materiales}

Para desarrollar los estímulos a aplicar en la versión final del experimento se realizó un estudio piloto con 30 alumnos de la misma facultad que no participaron de la versión final del experimento. En dicho examen se analizaron diferentes temas posibles de incorporar en el estudio a través de indagar su posición personal y percibida relevancia del asunto. A través de esas preguntas se buscaba obtener un estímulo que permitiera controlar adecuadamente el efecto de algunos factores observados en la literatura previa. Primero, se optó por un tema con una moderada relevancia. Ésta debía ser suficiente para involucrar a los participantes, aunque no alta como para que limite el efecto de la encuesta (Areni et al., 1998). Además, se eligió un tema que representara una posición dividida debido a que con eso se evita la influencia de un resultado cierto (Fleitas, 1971). A partir de ese pre-testeo, se desarrolló el estímulo final el cual indagó la opinión acerca de la preferencia por una semana de pruebas (opuesta al sistema de libre fijación de evaluaciones en los cursos). Ello se evaluó en base a dos preguntas que medían su preferencia. Una de ellas era de tres puntos y representaba las opciones "a favor", "en contra" e "indiferente". La segunda representaba una escala de 9 puntos en que 1 se refería a "muy fuertemente en contra" y 9 la opción opuesta, "muy fuertemente a favor". Estas dos escalas se correlacionaron altamente $(r=0.81)$, por lo que se sumaron y generaron una variable simple de preferencias que iba desde 2 a 12 (que se usó como variable dependiente).

Dicho tema fue presentado en cuadernillos que poseían ya sea una encuesta que favorecía o no la mantención de ese sistema de fijación de pruebas y en cada uno de ellos una versión en que los resultados eran moderadamente favoreciendo una u otra posición (54\% versus $46 \%$ ) o bien lo hacían de modo mucho más marcado (79\% versus $21 \%$ ). Esos porcentajes se usaron siguiendo un estudios previo que examinó la fuerza de la encuesta como variable relevante (Mehrabian, 1998).

Respecto del refinamiento de los test utilizados para medir las susceptibilidad individuales al efecto de las encuestas, se midió la validez interna del EPI y del test Need for Cognition. En el caso del primero, se puedo observar un coeficiente de Cronbach para la escala de introversión, extroversión y veracidad superior a 0.75 , lo cual hizo mantener el uso completo de éstas. En cuanto al segundo de los instrumentos, se obtuvo una coeficiente de 0.7 si se eliminaban dos preguntas, lo cual fue realizado para que la prueba tuviese suficiente consistencia interna al ser usada.

\section{Procedimiento}

El experimento se desarrolló en aplicaciones colectivas de aproximadamente 25 personas cada una. En dichas sesiones, a cada participante se le asignó aletoriamente una de las cuatro posibles combinaciones del estímulo, de modo que todos los participantes tuviesen la misma probabilidad de recibir cualquiera de las formas. A los estudiantes se les explicó que los resultados que encontrarían en el cuadernillo eran el resultado de un estudio que se realizó unos poco meses entes en la misma facultad. Luego de ser expuestos al estímulo, los alumnos debían expresar su preferencia ya sea a favor o en contra del tema preguntado (cambiar el sistema de semana de pruebas actualmente vigente). Finalmente, se les solicitaba que contestaran el Inventario de Eysenck y el test Need for Cognition.

\section{Resultados}

\section{Efecto de la Fuerza y la Dirección de la Encuesta}

El primer análisis de este estudio examinó si las variables independientes fuerza del resultado y dirección de éste tenían un efecto sobre la variable dependiente, representada por la manifestación de preferencia. Los datos de este estudio muestran que existe una interacción significativa, observándose un main effect sólo en la variable fuerza sobre la preferencia manifestada, con un $F=4.640$ y $p<0.05$. Al mirar los datos expuestos en la Figura 1, se puede detectar que las personas que recibieron la encuesta con una distancia fuerte entre las posiciones a favor y en contra ( $79 \%$ versus $21 \%$ ) se vieron influenciada en la manifestación de su preferencia. En este caso, en la escala de 2 a 12 que medía preferencias, el promedio de quienes recibieron la encuesta con mayor fuerza fue 8.41 en comparación con los 6.41 exhibidos por quienes recibieron la encuesta cuya fuerza era moderada ( $54 \%$ versus $46 \% ; t=1.6, p<$ $0.05)$. Al mirar las celdas expuestas en la tabla de la Figura 1, se puede apreciar que esta diferencia es principalmente causada por los casos en que la encuesta fue a favor, donde se apreciaron diferencias significativas en las medias detectadas para ambos niveles de fuerza (9.01 versus 5.94; $t=2.3, p<$ 
0.05). Esa diferencia no fue relevante en el caso de las encuestas en contra, donde la diferencia entre los valores observados (7.81 versus 6.88$)$ no fue estadísticamente significativa. En resumen se aprecian, en el grupo que vio encuestas marcadamente a favor de un grupo, un efecto en la dirección de lo propuesto por el efecto bandwagon.

Por su parte, la dirección de los resultados (a favor o en contra) no mostró producir un main effect significativo con un $F=0.238$ y $p=0.238$. De hecho los promedios residuales en la manifestación de preferencias fueron 7.38 y 7.48. Esto significa que el agregado total recibir una encuesta, privilegiando una u otra posición en el referéndum, no generó un efecto significativo en la variación de la preferencia (ver Figura 1). Algo similar ocurrió con el efecto conjunto de la dirección y la fuerza de los resultados de la encuesta. Su efecto no fue significativo en el test intersujetos, aunque estuvo próximo a la significancia $(F=3.076$ y $p=0.084)$.

\section{Análisis de la Susceptibilidad al Efecto \\ "Bandwagon” Según Diferencias Individuales}

A continuación se buscó determinar qué características tienen las personas que presentaron el efecto. Para eso, se analizaron las diferencias en la correlación fuerza/ preferencia en los casos que vieron una encuesta a favor $(N=66)$, ya que éstos mostraron una variabilidad mayor en el sentido de lo predicho por el efecto bandwagon. En ellos se hizo la mencionada correlación tanto a nivel de totales como de los subgrupos generados por el test de Eysenk, Need for Cognition y el análisis por género.

El efecto de la personalidad. En primer lugar se pudo observar que a nivel de totales, la preferencia estuvo correlacionada con la fuerza $(r=0.49, p$ $<0.05$ ). Ello muestra que en la medida que los participantes recibieron una encuesta con mayor diferencia entre una posición y otra (fuerte) y no más consensual (moderada), era más probable que se inclinarán a favor del resultado mostrado por la encuesta (bandwagon).

A continuación se realizaron las mismas correlaciones entre todas las dimensiones del test de Eysenk, Need for Cognition, así como de acuerdo al género de los participantes. Respecto del primero de los test, se correlacionó fuerza y preferencia entre quienes presentaban alto y bajo puntaje en la escala de extroversión (sobre y bajo la media). En este análisis sólo se observó que el efecto era significativo en el caso de los primeros. Es decir, se pudo observar que la correlación entre las personas con alto puntaje en extroversión resultó significativa $(r=0.45, p<0.05)$, lo cual no se dio en el caso de las personas con bajo puntaje en esta dimensión $(r$ $=0.37, p>0.05)$. Es decir, la escala extroversión estuvo asociada significativamente con la presencia del efecto bandwagon sólo en el caso de aquellos que presentaban más alto puntaje en ésta. Cuando el mismo análisis se realizó tomando la escala de neuroticismo, se observó algo similar. Es decir, la correlación con un puntaje alto en esta dimensión resultó significativa $(r=0.45, p<0.5)$, lo cual no se detectó entre quienes poseían un puntaje inferior a la media en esta dimensión $(r=0.31, p>0.05)$.

El efecto del estilo cognitivo. De acuerdo a lo señalado por Areni et al. (2000), se separó a la muestra según el $35 \%$ más alto en puntaje, es decir, los que utilizaban más su ruta central, y el $35 \%$ que tenía el puntaje más bajo de la muestra, es decir, aquellos que utilizaban su ruta periférica. Luego de analizar la correlación entre las variables de fuerza y preferencia, se pudo ver que en ambos casos no hubo efecto significativo, es decir, la variable de cognición no sería determinante ni discriminante a la hora de saber si una persona puede presentar efecto bandwagon.

El efecto de la variable género. Para ver si el género fue determinante entre quienes presentaron efecto bandwagon, se realizó la correlación entre las variables fuerza y preferencia para ambos géneros. Se encontró una correlación significativa sólo en el caso de los hombres $(r=0.47, p<0.05)$ y no para las mujeres $(0.44, p<0.05)$. Esto significa que en este caso, el ser hombre aumenta la probabilidad de ser afectado por el efecto bandwagon.

\section{Discusión}

El presente estudio ha presentado una revisión acerca de la evidencia del efecto de las encuestas en las preferencias de las personas y un estudio experimental que busca expandir hallazgos previos en el área reportados por Mehrabian (1998). En concreto, se desarrolló un experimento que mostró la existencia del efecto bandwagon (aumentando significativamente la preferencia por un asunto en la misma dirección de la encuesta) en el caso de aquellas personas que vieron un estudio de opinión cuyos resultados presentaban altas diferencias entre una y otra posición (Glynn, Ostman \& McDonald, 1995; Mutz, 1998). 
Adicionalmente, el estudio mostró la existencia de diferencias individuales en términos de la susceptibilidad del presentar este efecto. Por un lado, se pudo detectar que el cambio en aquellas personas expuestas a encuestas con alta diferencia entre una posición y otra estaba significativamente asociado con un alto puntaje en las escalas de extroversión y neoroticismo. Ello no debiese sorprender del todo, no sólo por la proximidad con lo observado por Mehrabian (1998) anteriormente, sino también por una larga data de investigaciones en áreas aplicadas de la psicología como es la conducta del consumidor y la persuasión, donde estas dos dimensiones vinculadas con la apertura social y la mayor disposición al cambio han sido relacionadas con la influencia interpersonal y, en general, a responsividad frente a estímulos externos. De hecho un interesante estudio de Mooradian (1996) muestra estas mismas dos dimensiones como principales predictores de respuestas emocionales frente a la publicidad y al cambio en las actitudes hacia los productos allí promocionados (ver también Duhachek \& Iacobucci, 2005; Kassarjian, 1971; Nairn \& Berthon, 2003).
En lo que se refiere específicamente a la relevancia de la extroversión, resulta interesante plantear que la mayor dependencia y apertura social que poseería este grupo, debería vincularse con el hecho que para ellos la opinión grupal (expresada en un estudio de opinión) puede representar una clave significativa de interpretación del mundo (Eysenck, 1991). Ello es particularmente relevante cuando se habla de encuestas que presentan resultados bastante radicales y no moderados, lo cual podría producir un cuestionamiento acerca de las creencias personales más fuerte entre aquellos que poseen una mayor apertura al exterior y que otorgan al medio un rol proveedor de aprendizajes mayor (Koole, Jager, van den Berg, Vlek \& Hofstee, 2001).

En la relación particular entre una mayor presencia del efecto bandwagon asociado a puntajes altos de neuroticismo, debería ser interpretado en la línea de que se trata de una mayor inestabilidad y por tanto más cambiante. Ello asociado a que también se asocia a personalidades con una alta apertura al exterior pero más bien en la línea de obtener aceptación a través de adaptarse a las expectativas

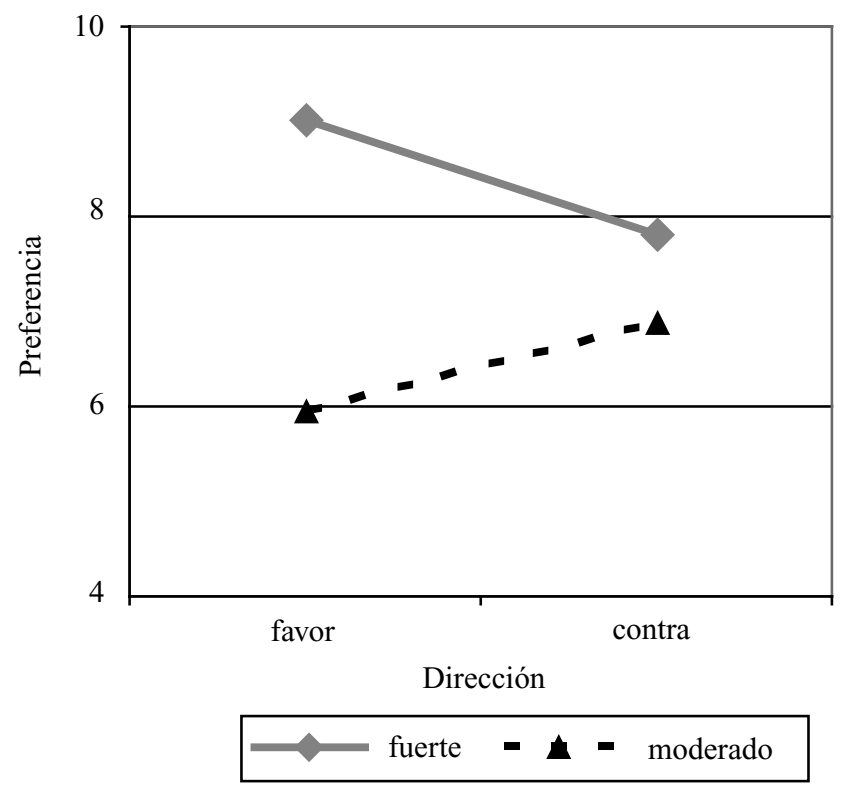

\begin{tabular}{lccc}
\hline & A Favor & En Contra & Residuales \\
\hline Fuertemente & 9.01 & 7.81 & 8.41 \\
Moderadamente & 5.94 & 6.88 & 6.41 \\
Residuales & 7.47 & 7.34 & \\
\hline
\end{tabular}

Figura 1. Efecto de la dirección y dirección de la encuesta sobre la manifestación de preferencia. 
que posee el entorno que le rodea. En este sentido, un puntaje más alto en neuroticismo puede tender a ocultar e incluso modificar su opinión original y manifestar en cambio aquella socialmente dominante (Katz, Blumer \& Gurevitch, 1974). Así, un grupo lábil y abierto socialmente aparece en este caso respondiendo de modo más fuerte a la presencia de una encuesta con alta diferencia entre las posiciones (Mehrabian, 1998).

En cuanto al estilo cognitivo, se apreció que no existían diferencias entre las personas con tendencia al uso de una ruta más periférica o centralizada en relación al efecto de las encuestas sobre la manifestación de sus preferencias. Esto pareciera estar mostrando que las encuestas no parecen ser un elemento que per se sea un estímulo más significativo para un grupo u otro. Al contrario, esto debiese ser leído más en la línea de lo planteado por Areni et al. (2000). Dichos autores sugieren que un estudio de opinión puede ser un elemento persuasivo tanto al ser procesado por personas que usan preferentemente la ruta central o periférica.

Finalmente, el análisis por género mostró que los hombres tuvieron una influencia significativa de los estudios de opinión y no así las mujeres. Estos resultados contradicen los hallazgos previos de Mehrabian (1998), quien había observado la situación opuesta. De esta forma, se abre la puerta a la necesidad de desarrollar nueva investigación que permita ahondar más en detalle en el rol del género como mediador en la influencias de los estudios de opinión en particular y de la persuasión en general.

En su conjunto, estos resultados muestran la relevancia que adquiere la forma y estructura en que se presenta la encuesta en relación a su persuasividad, así como las variables individuales, en este caso la personalidad y el género de quien la recibe. Este estudio destaca que la forma de presentación, en términos de cuán grande es el gap entre las posiciones puede ser un elemento clave a la hora de generar un cambio actitudinal cuando se desarrolla una estrategia comunicacional. En relación a las audiencias, se debiese pensar que segmentos sociales más atentos a claves externas podrían ser más persuadibles, particularmente cuando una encuesta representa tendencias claras.

Finalmente, es importante destacar las limitaciones de este estudio. Por un lado, presenta un tipo de estudios de opinión (referidos a un referéndum y no a una encuesta), lo que hace sus alcances limitados. Adicionalmente, se examinó este asunto con personas de alto nivel educacional (universitarios), lo cual genera otro sesgo respecto de lo que ocurre en el país, donde según las cifras oficiales Chile posee un nivel educacional promedio de 9.9 años de su fuerza laboral, y los universitarios superan todos los 12 años (UNESCO, 2003). Tercero, se utilizó un diseño experimental. Si bien se trata de la forma más aceptada para desarrollar estudios de causalidad, también ha sido recurrentemente criticado por la validez ecológica de sus resultados (Rosenthal \& Rosnow, 2005). Finalmente, se examinó un asunto de alcance académico y de la vida diaria de los entrevistados. Ello significa que existía un grado de impacto de la información directo, lo cual no necesariamente es así en las elecciones o referéndums sociales.

\section{Referencias}

Andersen, R. (2000). Reporting public opinion polls: The media and the 1997 Canadian election. International Journal of Public Opinion Research, 12(3), 285-298.

Ansolabehere, S. \& Iyengar, S. (1993). Information and electoral attitudes: A case of judgment under uncertainty. En S. Iyengar \& W. J. McGuire (Eds.), Explorations in political psychology (pp. 321-337). Durham: Duke University Press.

Areni C., Ferrell, M. \& Wilcox, J. (1998). The influence of poll results on public opinion: Examining the personal consequences of the poll topic. Journal of Nonprofit and Voluntary Sector Marketing, 3(4), 298-311.

Areni, C., Ferrell, M. \& Wilcox, J. (2000). The persuasive impact of reported group opinions on individuals low vs. high in Need for Cognition: Rationalization vs. biased elaboration? Journal of Psychology and Marketing, 17, 855-877.

Bartels, L. M. (1985). Expectations and preferences in presidential nominating campaigns. American Political Science Review, 78(4), 804-815.

Ceci, S. J. \& Kain, E. L. (1982). Jumping on the bandwagon with the underdog: The impact of attitude polls on polling behavior. Public Opinion Quarterly, 46, 228-242.

Cialdini, R. B. (1993). Influence: Science and practice. New York: Harper Collins.

Cloutier, E., Nadeau, R. \& Guay, J. (1989). Bandwagoning and underdogging on North American free trade: A quasi-experimental panel study of opinion movement. International Journal of Public Opinion Research, 1(3), 206-220.

Crespin, M. \& Wielen, R. J. (2002, abril). The influence of media projections on voter turnout in presidential elections from 1980-2000. Ponencia presentada en el Annual Meeting of the Midwest Political Science Association, Chicago, Illinois.

Diaz-Castillo, L. (2005). Bandwagon and underdog effects on a low-information, low-involvement election. Ann Arbor: Proquest/ UMI.

Donsbach, W. (2001). Who's afraid of election polls? Normative and empirical arguments for the freedom of pre-election surveys. Ponencia presentada en el Congreso ESOMAR, Ámsterdam, Holanda.

Duhachek, A. \& Iacobucci, D. (2005). Consumer personality and coping: Testing rival theories of process. Journal of Consumer Psychology, 15(1), 52-63.

Eysenck, H. J. (1990). Biological dimensions of personality. En L. 
A. Pervin (Ed.), Handbook of personality: Theory and research (pp. 244-276). Nueva York: Guilford Press.

Eysenck, H. J. (1991). Dimensions of personality: 16, 5 or 3 ? Criteria for taxonomic paradigm. Personality and Individual Differences, 12, 773-790.

Eysenck, H. J. (1992). The definition and measurement of psychoticism. Personality and Individual Differences, 13, 757-785.

Eysenck, H. \& Eysenck, S. (1964). The Manual of the Eysenck Personality Inventory. EPI. Londres: University of London Press.

Fleitas, D. W. (1971). Bandwagon and underdog effects in minimal information elections. American Political Science Review, 65, 434-438.

Gartner, M. (1976). Endogenous bandwagon and underdog effects in a rational choice model. Public Choice, 25(1), 83-139.

Glynn, C. J., Ostman, R. E. \& Mc Donald, D. G. (1995). Opinions, perception, and social reality. En T. L. Glasser \& C. T. Salmon (Eds.), Public opinion and the communication of consent (pp. 249-280). Nueva York: The Guilford Press.

Goidel, R. K. \& Shields, T. G. (1996). The vanishing marginals, the bandwagon and the mass media. The Journal of Politics, $56,802-810$.

Haugtvedt, C. P., Petty, R. E. \& Cacioppo, J. T. (1992). Need for Cognition \& advertising: Understanding the role of personality variables in consumer behavior. Journal of Consumer Behavior, 3(1), 239-260.

Kaplowitz, S. A., Fink, E. L., D’Alessio D. \& Armstrong, G. B. (1983). Anonymity, strength of attitude and the influence of public opinion polls. Human Communication Research, 10(1), 5-25

Kassarjian, H. H. (1971). Personality and consumer behavior: A review. Journal of Marketing Research, 8(4), 409-418.

Katz, E. \& Lazarsfeld, P. (1955). Personal influence, New York: The Free Press.

Katz, E., Blumer, J. \& Gurevitch, M. (1974). Utilization of mass by the individual. En J. Blumer \& E. Katz (Eds.), The Uses communications (pp. 19-32). Beverly Hills, CA: Sage.

Koole, S. L., Jager, W., Van den Berg, A. E., Vlek, C. A. J. \& Hofstee, W. K. (2001). On the social nature of personality: Effects of extraversion, agreeableness, and feedback about collective resource use on cooperation in a resource dilemma. Personality and Social Psychology Bulletin, 27(3), 289-301.

Lachapelle, G. (1991). Polls and the media in Canadian elections: Taking the pulse. Royal Commission on Electoral Reform and Party Financing (Volume 16). Toronto: Dundurn Press.

Lang, K. \& Lang, G. E. (1984). The impact of polls in public opinion. The Annals of the American Academy of Political and Social Sciences, 472, 129-142.

Lavrakas, P. J., Holley, J. K. \& Miller, P. V. (1991). Public reactions to polling news during the 1988 presidential election campaign. En P. J. Lavrakas \& J. K. Holley (Eds.), Polling and Presidential election coverage (pp. 151-183). Newbury Park, CA: Sage.

Lavrakas, P. J. and Traugott, M. W. (2000). Election polls, the news media and democracy. New York: Chatham House Publishers.

Maarek, P. (1997). Marketing político y comunicación. Claves para una buena información política. Barcelona: Paidós.

Marsh, C. (1984). Back on the bandwagon: The effect of opinion polls on public opinion. British Journal of Political Science,
15(1), 51-74.

McAllister, I. \& Studlar, D. T. (1991). Bandwagon, underdog, or projection? Opinion polls and electoral choice in Britain, 1979-1987. Journal of Politics, 53, 720-741.

McQuail, D. (2000). Mass communication theory. Londres: Sage.

Mehrabian, A. (1998). Effects of pool reports on voter preference. Journal of Applied Social Psychology, 23, 2119-2130.

Mendelson, H. (1966). Election-day broadcasts and terminal voting decisions. Public Opinion Quarterly, 30, 212-225.

Mooradian, T. A. (1996). Personality and ad-evoked feelings: The case for extraversion and neuroticism. Journal of the Academy of Marketing Science, 24(2), 99-109.

Morwitz, V. G. \& Pluzinski, C. (1996). Do polls reflect opinions or do opinions reflect polls? The impact of political polling on voters' expectations, preferences, and behavior. Journal of Consumer Research, 23(1), 53-67.

Mutz, D. C. (1998). Impersonal influence. Cambridge: Cambridge University Press.

Nairn, A. \& Berthon, P. (2003). Creating the customer: The influence of advertising on consumer market segments - Evidence and ethics. Journal of Business Ethics, 42(1), 83-100.

Navazio, R. (1977). An experimental approach to bandwagon research. Public Opinion Quarterly, 41(2), 217-225.

Noelle-Neumann, E. (1984). The spiral of silence: Public opinion - Our social skin. Chicago: University of Chicago.

Petty, R. E. \& Cacioppo, J. T. (1986a). The elaboration Likelihood Model of persuasion. New York: Academic Press.

Petty, R. E. \& Cacioppo, J. T. (1986b). Communication and persuasion: Central and peripheral routes to attitude change. New York: SpringerVerlag.

Rosenthal, R. \& Rosnow, R. L. (2005). Beginning behavioral research: A conceptual primer (5th Ed.). Upper Saddle River, NJ: Prentice Hall.

Roshwalb, I. \& Resnicoff L. (1971). The impact of endorsements and published polls in the 1970 New York senatorial election. Public Opinion Quarterly, 35(3), 410-414.

Simon, H. (1954). Bandwagon and underdog effects and the possibility of election predictions. Public Opinion Quarterly, 18(3), 245-253.

Schmitt-Beck, R. (1996). Mass media, the electorate and the bandwagon: A study of communication effects on vote choice in Germany. International Journal of Public Opinion Research, $8,266-291$.

Sudman, S. (1986). Do exit polls influence voting behavior? Public Opinion Quarterly, 50(3), 331-339.

Tangeras, T. P. (1998). On the role of opinion polls in political competition. Institute for International Economic Studies, 655. Recuperado el 10 de marzo de 2006, desde http://www.iies. su.se/publications/seminarpapers/655.pdf

UNESCO. (2003). Tercer informe WEI: Financiamiento de la educación. Santiago: Autor.

West, D. (1991). Polling effects on election campaigns. Political Behavior, 13, 151-163.

White, G. M. (1975). Contextual determinants of opinion judgments: Field experimental probes of judgmental relativity boundary conditions. Journal of Personality and Social Psychology, 32, 1047-1054.

Wolf, M. (1994). Los efectos sociales de los media. Barcelona: Paidós.

Fecha de recepción: septiembre de 2006.

Fecha de aceptación: junio de 2007. 